Cómo citar este trabajo: Iglesias Skulj, A. (20XX). Estrategias de silenciamiento de Ixs trabajadorxs sexuales en Argentina: Políticas y discursos expertos. Revista del Laboratorio Iberoamericano para el Estudio Sociohistórico de las Sexualidades, 04 art. 13, 252-370. https://doi.org/10.46661/ relies.4994

\title{
Estrategias de silenciamiento de Ixs trabajadorxs sexuales en Argentina: Políticas y discursos expertos
}

\author{
Strategies for silencing sex workers in Argentina: Policies and expert \\ discourses
}

\begin{abstract}
Agustina Iglesias Skulj
Lcda. en Derecho (Universidad de Buenos Aires). Doctora en Derecho Penal (Universidad de Salamanca). Criminóloga

Transfeminista e Investigadora Independiente. Integrante de FUERTSA (Frente de Unidad Emancipatorio por el Reconocimiento de los Derechos de Trabajadorxs Sexuales en Argentina)

fermelita@gmail.com
\end{abstract}

\section{Resumen}

En el ámbito de la ley, el cuerpo sexuado de las mujeres -cis, trans y travestis-está atravesado por una multiplicidad de elementos y categorías -delincuente, reproductor, transgresor, victimizadoque organizan determinadas relaciones de poder y cuestionan la pretendida univocidad del ordenamiento jurídico. Por lo tanto, en el ámbito de las políticas anti-trata no alcanza con criticar las prohibiciones legales, sino que es necesario analizarlas como una expresión que produce relaciones de poder provisionales y contradictorias. En particular en este texto, se abordarán los métodos de silenciamiento del (neo)abolicionismo frente a las denuncias de Ixs trabajadorxs del sexo acerca de los efectos materiales y simbólicos de las políticas anti-trata y la penalización del cliente de servicios sexuales.

Palabras clave: Trata Sexual, Vulnerabilidad, Modelo Sueco, Empoderamiento Punitivo. 


\begin{abstract}
In the field of law, the sexed body of women - cis and trans and transvestites - is made up of a multiplicity of elements and categories - criminal, reproductive, transgressor, victimized - that organize power relations and question the alleged univocity of the legal system. It is not enough just to criticize the legal prohibitions, but it is necessary to analyze them as an expression that produces provisional and contradictory power relations. In particular in this text, the methods of silencing by (neo)aboliionist will be addressed in the face of complaints by sex workers about the material and symbolic effects of anti-trafficking policies and the criminalization of the client.
\end{abstract}

Key words: Sex Trafficking, Vulnerability, Swedish Model, Penal Empowerment. 


\section{Introducción}

Prostitución sí, prostitución no. Trabajo sexual sí, trabajo sexual no. Estos son los términos en los que habitualmente se propone el debate y su rigidez impide que podamos pensar más allá de lanzarnos acusaciones de uno y otro lado. Como en otras cuestiones contemporáneas, parece que lo importante es sentar una posición clara y definitiva.

Ese maniqueísmo se traslada a los análisis de la intervención estatal en el comercio sexual, lo que se conoce como los modelos legales. A muy grosso modo, podemos describirlos así: el prohibicionismo -se sanciona a la persona que ofrece los servicios sexuales abarcando todos los intercambios-, el reglamentarismo -un régimen disciplinario que establece controles médicos y administrativos a la prostituta con la finalidad, por ejemplo, de evitar la transmisión de enfermedades venéreas-, el abolicionismo -que surge en respuesta al reglamentarismo- y la legalización -estrategias de descriminalización y reconocimiento de derechos laborales para las trabajadoras sexuales-. El abolicionismo considera que la prostitución es degradante en sí misma, por eso lucha activamente en contra de la legalización; sin embargo, de la mano de este "modelo" se ha ido capilarizando el control de la prostitución, a partir de ampliar los procesos de criminalización sobre las actividades de las personas involucradas desde las políticas que luchan contra la trata sexual de mujeres.

Los modelos citados habitualmente son utilizados para describir distintas etapas históricas en diversos países, pero no permiten dar cuenta de lo que sucede más allá de la rigidez del pensamiento jurídico que organiza la realidad entre "legal" o "ilegal". Esta clase de análisis impide por ejemplo que podamos incluir los cambios socioculturales y económicos que se han producido en la concepción de la sexualidad y en el desarrollo del mercado sexual.

A lo anterior debe agregarse que el análisis de los efectos materiales de cualquiera de los modelos legales en el mercado sexual se suplanta generalmente por un fetichismo legal que, la mayor parte de las veces, tiene el efecto de acallar las voces de quienes están involucradas y erradicar la multiplicidad de experiencias vitales que se producen en los distintos tipos de mercados sexuales, invisibiliza los intereses que hay detrás de estas legislaciones y silencia la multiplicidad de las voces sobre la cuestión. Negar las tensiones normativas que se dan entre lo supra/inter/nacional y su aplicación concreta, y los efectos que generan en los distintos lugares solo promueve la ampliación de los procesos de criminalización en el contexto de las políticas anti-trata en el ámbito local.

No obstante, ese fetichismo también ha funcionado para la conclusión opuesta. Se tiende a pensar que cualquiera de los modelos legales produce los mismos efectos en el momento de ser implementados, aunque esto no sea así (Scoular y O’Neill, 2007).

En el ámbito de la ley, el cuerpo sexuado de las mujeres -cis y trans y travestis-está constituido por una multiplicidad de elementos y categorías - delincuente, reproductor, transgresor, victimizadoque organizan determinadas relaciones de poder y cuestionan la pretendida univocidad del ordenamiento jurídico. No basta sólo con criticar las prohibiciones legales, sino que es necesario analizarlas como parte de un campo que produce relaciones de poder provisionales $y$ contradictorias.

Por lo tanto, la propuesta de esta contribución se dirige a analizar los efectos que ha producido el "Modelo Sueco" intentando escapar de los análisis criticados más arriba. Para ello, recuperamos la expresión (neo)abolicionismo para hacer referencia no solamente a una cuestión temporal desde la 
sanción del Protocolo de Palermo contra la trata ${ }^{1}$, como vector de diferenciación con las anteriores campañas contra la trata de blancas, sino a una particular forma de gobierno de la prostitución, que retoma elementos de la criminología positivista como método para individualizar las condiciones estructurales de los mercados de trabajo cada vez más informalizados y precarizados. El prefijo (neo) pretende marcar una distinción, y también las continuidades, con el movimiento abolicionista de la prostitución en el contexto de la lucha contra la trata de blancas que se dio entre fines del S. XIX y comienzos del s. XX en Inglaterra y Francia. Si bien en la actualidad permanece el objetivo de acabar con la prostitución, con la emergencia del Modelo Sueco lo que se busca es desalentar la demanda de servicios sexuales, bajo la consideración de que esta es una herramienta más eficaz en la lucha contra la trata con fines de explotación sexual; asimismo, esa individualización funciona para abstraer la explotación en el ámbito de la trata de los regímenes de informalización y precarización de los mercados de trabajo, sobre todo para el caso de mujeres (cis, trans y travestis) migrantes.

Como estrategia para salir del atolladero de los análisis dedicados a los modelos legales, optamos por visiblizar las denuncias de Ixs trabajadorxs del sexo acerca de los efectos materiales y simbólicos de las políticas anti-trata $y$, en particular, las campañas en dicho contexto que apuntan a la penalización del cliente de servicios sexuales.

Con este objetivo, analizaremos el (neo)abolicionismo como un dispositivo ${ }^{2}$, dado que brinda la posibilidad de desentrañar las racionalidades actuales que permiten que patriarcado, vulnerabilidad y explotación sean instrumentalizados para aumentar la extensión y la intensidad de las tecnologías de control sobre las cis, trans mujeres y travestis ${ }^{3}$ bajo la gobernanza feminista de la trata sexual (Halley et.al., 2006).

El término de gobernanza feminista fue acuñado para hacer referencia a los procesos a través de los cuales las reivindicaciones feministas han logrado instalarse en las instituciones nacionales e

${ }^{1}$ Este instrumento se sancionó en 2000 y es complementario de la Convención de Naciones Unidas contra la Criminalidad Organizada Transnacional y tiene por objeto prevenir, reprimir y sancionar la trata de personas, especialmente niñxs y mujeres. Asimismo, en aquella oportunidad se sancionó Protocolo Contra el Tráfico llícito de Migrantes por Tierra, Mar y Aire. En el ámbito de la redacción del Protocolo contra la trata, se produjeron acaloradas discusiones para arribar a una definición del delito que lograse superar los marcos históricos que la identificaban, sin ulteriores matices, con el ejercicio de la prostitución. Con todo, en este texto la expresión (neo)abolicionismo no sólo remite a una cuestión temporal como vector de diferenciación con las anteriores campañas contra la trata de blancas, sino a una particular forma de gobierno de la prostitución que retoma elementos de la criminología positivista como método para individualizar las condiciones estructurales de los mercados de trabajo cada vez más informalizados y precarizados. EI prefijo (neo) pretende marcar una distinción, y también las continuidades, con el movimiento abolicionista de la prostitución en el contexto de la lucha contra la trata de blancas a caballo entre fines del S. XIX y comienzos del s. XX en Inglaterra y Francia. Si bien permanece el objetivo de acabar con la prostitución, desde la emergencia del Modelo Sueco lo que se busca es desalentar la demanda de servicios sexuales como un método eficaz para cumplir aquél objetivo. Como será explicado en lo que sigue, este modelo establece una diferenciación de las distintas modalidades de explotación en el contexto de la trata de personas, dando prioridad a la finalidad de explotación sexual, equiparada al ejercicio de la prostitución, abstrayéndola de condicionamientos sociales o de las políticas de reducción de daños y optando por la criminalización de la demanda.

2 Foucault (1984) define al dispositivo como un conjunto heterogéneo que comprende discursos, instituciones, instalaciones arquitectónicas, decisiones reglamentarias, leyes, medidas administrativas, enunciados científicos, proposiciones filosóficas, morales, filantrópicas; más precisamente el dispositivo es el entramado que describen estos elementos. Una de las funciones del dispositivo es que permite justificar y ocultar una práctica, dando acceso a un nuevo campo de gubernamentalidad.

${ }^{3}$ La distinción es pertinente en tanto y en cuanto podemos interpretar extensivamente los sujetos de protección del Protocolo contra la trata. Por tanto, en el texto resulta necesario distinguir entre las cismujeres, es decir, aquellas corporalidades en las que coincide el género biológico con la identidad de género para señalar los efectos diferenciados con aquellxs que se perciben trans o travestis.

Revista del Laboratorio Iberoamericano para el Estudio Sociohistórico de las Sexualidades https://doi.org/10.46661/relies.4994 
internacionales. Este feminismo despliega una estrategia fragmentada y dispersa en el ámbito del derecho incidiendo no solo en las formas de legislar, de litigar o de diseñar políticas públicas, sino también en las campañas para la toma de conciencia respecto de problemáticas que atañen a las mujeres. Halley señala que estas estrategias van de la mano del feminismo legal estadounidense que desde los años noventa del siglo XX ha teorizado recurrentemente sobre la ley penal y las formas de control social, insertándose sin mayores dificultades en el "giro punitivo" denunciado por la literatura criminológica estadounidense y latinoamericana desde finales de los años ochenta, y más recientemente, por las críticas al devenir punitivo del feminismo. Las políticas de lucha contra la trata sexual son paradigmáticas en este sentido.

\section{El (neo)Abolicionismo y sus fetiches normativos}

En particular, nos interesa analizar al Modelo Sueco -que propone la criminalización del clientecomo parte sustancial de la propuesta (neo)abolicionista. Su positivización en ese país en 1999 se produjo a partir de una reforma del Código Penal que buscó eliminar la práctica masculina de comprar servicios sexuales, dejando por fuera del alcance de la prohibición al ofrecimiento. Dentro de las finalidades enunciadas por Ixs legisladorxs en aquel momento estaba la de estigmatizar al varón que demandaba este tipo de servicios. Paralelamente, esta ley estaba orientada a que las trabajadoras sexuales -entre 1.850 a 3.000, según las estimaciones al momento de presentar el proyecto de ley-abandonaran el trabajo sexual. La modificación en la legislación penal fue considerada una medida eficaz para acabar con la trata sexual de mujeres, fundamentalmente migrantes e irregularizadas (Dedillet y Östergren, 2012; Ekberg, 2004).

Entre las múltiples críticas que ha recibido este modelo nos interesa poner de manifiesto aquellas que apuntan al contexto político en el cual esta propuesta del feminismo institucionalizado logró los apoyos necesarios para la sanción de la ley, con independencia de los intereses comunes que pudieran tener los diferentes actores. Tal como sucedió con la trata de blancas a principios de siglo pasado (Andrijasevic, 2010:41), la atmósfera condensaba los temores a la pérdida de la identidad nacional, que rondaron la incorporación de Suecia a la Unión Europea (UE). Kulick (2003: 330) describe el proceso a través del cual la lucha contra la prostitución y la trata ofició como terreno fértil de las discusiones acerca del tipo de relación que establecería Suecia con la UE ${ }^{4}$ (Kulick, 2003: 220).

En el ámbito de Naciones Unidas, contemporáneamente a la sanción de la criminalización de la demanda, se estaba discutiendo la Convención contra la Criminalidad Organizada Transnacional y sus dos protocolos complementarios. Uno de ellos está específicamente dedicado a la trata de personas enmarcado en un lenguaje de derechos humanos frente a este tipo de delincuencia. Junto al tráfico de migrantes clandestinizados, el terrorismo, o el tráfico de drogas o armas, la trata se problematizó como una amenaza transnacional al imperio de la ley (Aradau 2004, 2008).

En este sentido, Claudia Aradau (2004:254) señala que el Protocolo es un instrumento paradigmático de las relaciones entre los discursos securitarios y de protección de derechos humanos, y que la supuesta incompatibilidad entre ellos se desarma mediante las intervenciones promovidas sobre la conducta de colectivos y de las víctimas individualizadas. Según Aradau, el discurso humanitario funciona a partir de lo que ella definió como "políticas de la compasión" donde

\footnotetext{
${ }^{4}$ El proceso de sanción de la ley propuesta en 1998 por el feminismo sueco estuvo signado por el miedo a la inmigración proveniente del este europeo, que estaba siendo identificada con varios tipos de la criminalidad organizada, y en el caso de las mujeres, con la prostitución. Los medios de comunicación suecos alertaron sobre los peligros de la delincuencia y expresaron su temor respecto de un lobby pro-prostitución expandiéndose por Europa. A ambas cuestiones las asimilaban a una penetración de las fronteras del Estado nación.
}

Revista del Laboratorio Iberoamericano para el Estudio https://doi.org/10.46661/relies.4994

Sociohistórico de las Sexualidades 
las intervenciones son moldeadas a través de las emociones que despierta la víctima y, éstas a su vez, se articulan con las "políticas del riesgo" que intervienen mediante el despliegue de tecnologías de gestión actuariales. Algunos análisis sobre los procesos de securitización consideran que este instrumento legal forma parte del cambio de la finalidad de los programas de las Naciones Unidas para combatir el subdesarrollo y que, en la actualidad, son problematizados como "seguridad

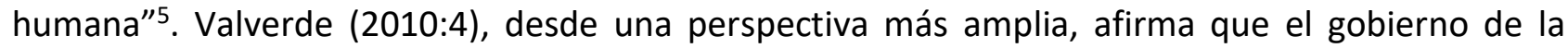
seguridad significa algo más que poner en marcha ciertas medidas con el objetivo de defender bienes o personas ya existentes, puesto que crea nuevas esferas de gobernanza e impide que la definición de seguridad sea algo estático y, por el contrario, debe servirnos para detectar los movimientos y contradicciones emergentes en esas nuevas esferas.

Por lo tanto, el gobierno de la trata sexual como parte de la gobernanza feminista, promueve que un mayor número de conflictos relativos a la seguridad de las mujeres se traduzca en una intervención excepcional, en el aumento de las penas y/o en la eficacia de las fuerzas de seguridad para combatir esa emergencia (Bumiller, 2008:114; Scoular, 2004:345). A mayor abundamiento, las definiciones (neo)abolicionistas de la prostitución fueron fácilmente instrumentalizadas por un "modelo legal" que parte de una concepción esencialista de la violencia, cuyo resultado más inmediato se advierte en la criminalización del trabajo sexual en sus múltiples expresiones, fundamentalmente en cuanto éste logra articular alguna forma de organización estable.

Asimismo, este dispositivo se insertó sin mayores dificultades en el entramado de las tecnologías de control de las poblaciones peligrosas y de los individuos, propios del control de la prostitución desde finales del S. XIX; no obstante, en la actualidad, esos controles son modulados a través de la categoría de riesgo en los procesos de selectividad de las fuerzas de seguridad y para la confección de los perfiles de victimización de este delito.

La trata sexual inserta en el lenguaje punitivo se presenta como una cuestión simplificada de castigo a los tratantes y el rescate de las víctimas, ambxs con perfiles racializados (Kotiswaran, 2014). Esta mirada dicotómica fue denominada "enfoque trafiquista" (Agustin, 2007) y es el resultado del lenguaje excepcional y homogéneo que genera nuevas jerarquizaciones y formas de inclusión/exclusión de lxs sujetxs amenazadxs (Aradau, 2008) en este ámbito.

\section{Los laberintos de las (in)definiciones legales}

En la abundante literatura dedicada al estudio de las políticas contra la trata sexual de cismujeres encontramos diversos intentos de confeccionar las herramientas analíticas capaces de mitigar el carácter ambiguo de la definición legal del Protocolo, y su traducción igualmente deficitaria a los ordenamientos jurídico-penales locales. En este sentido, Kelly (2005) afirma que esa característica permite que este delito sea potencialmente utilizado para criminalizar un amplio abanico de situaciones y conductas. Su propuesta, por tanto, junto a la de muchxs otrxs autorxs, está dirigida a establecer una distinción que inhabilite la identificación entre la prostitución voluntaria y la forzada. Esta identificación ha dado lugar a una profusa literatura crítica respecto de los procesos de criminalización desplegados por las políticas criminales que han sido diseñadas para luchar contra la explotación sexual (Bernstein, 2012; Hoang y Parreñas, 2014; Sanders, O’Neill y Pitcher, 2009; Saunders y Soderlund, 2003; Varela, 2013; Weitzer 2011; Daich y Varela AMMAR/RedTraSex, 2014; 2016; Amnesty, 2016).

\footnotetext{
${ }^{5}$ En el ámbito de la ONU, la Comisión de Seguridad Humana se estableció en 2001 como respuesta la llamada en 2000 por parte de la Secretaría General de un mundo libre de miedo.
}

Revista del Laboratorio Iberoamericano para el Estudio https://doi.org/10.46661/relies.4994

Sociohistórico de las Sexualidades 
Tal como quedó plasmado el delito en Argentina recogiendo los lineamientos del Protocolo, no es posible distinguir entre una situación de explotación sexual que configure dicha conducta y la prostitución ejercida voluntariamente (Iglesias Skulj, 2013:284).

En esta oportunidad, nuestra propuesta consiste en incorporar dicha ambigüedad al análisis, puesto que abre líneas de problematización más productivas que intentar encajar estas prácticas en un modelo legal (Agustín, 2007; Munro y Scoular, 2012).

Incorporar la ambigüedad al análisis nos permite visibilizar los efectos materiales derivados de la campaña "sin clientes no hay trata", si bien los diferentes intentos de crear una figura penal específica para criminalizar la demanda de servicios sexuales no lograron convertirse en derecho positivo en Argentina; de hecho, se encuentran vigentes legislaciones -tanto a nivel local y nacionalque habilitarían la persecución de tales conductas aunque no se registra en la jurisprudencia ninguna causa dirigida al castigo del cliente.

No obstante, el fuerte carácter simbólico de la criminalización del cliente de servicios sexuales ha sido instrumentalizado en las campañas contra la explotación sexual a partir del año 2010 en Argentina. Zaida Gatti, titular del Programa Nacional de Rescate y Acompañamiento de las personas damnificadas por el delito de trata7 sostuvo que: "Pero, así como puede afirmarse que una de las principales causas de la Trata es que las víctimas frecuentemente proceden de clases económicas desfavorecidas, otra de las raíces del problema, que no podemos dejar de visualizar, es la demanda, o sea el cliente o usuario. Recordamos que: sin clientes no hay trata. En el circuito de oferta y demanda, quien es prostituida no obtiene ninguna ganancia. Las prácticas sexistas son llevadas a su límite porque el supuesto intercambio jamás es equitativo: lejos de ser un acuerdo entre pares, las mujeres son ubicadas en un lugar de subordinación y son despojadas tanto de su poder de decisión como de su autonomía. Los clientes creen contar con derecho de propiedad" (Gatti, 2013:9).

Estas declaraciones dan cuenta de cómo se diseñaron las políticas anti-trata y cómo se ampliaron los procesos de criminalización y estigmatización contra el trabajo sexual ${ }^{8}$, además del fuerte

\footnotetext{
${ }^{6}$ En 2008 se sancionó la ley 26.364 que introdujo la tipificación del delito de trata de personas en el Art. 145 bis que estableció una "pena de tres a seis años de prisión a quien captare, transportare o trasladare, dentro del país o hacia el exterior, acogiere o recibiere personas mayores de 18 años de edad, cuando mediare engaño, fraude, violencia, amenaza o cualquier otro medio de intimidación o coerción, abuso de autoridad o de una situación de vulnerabilidad, concesión o recepción de pagos o beneficios para obtener el consentimiento de una persona que tenga autoridad sobre una víctima, con fines de explotación". En los casos de tratarse de personas menores de edad, tal como preveía el art. 145 ter. la redacción se mantenía con prescindencia de los medios comisivos, puesto que estos sujetos no pueden prestar consentimiento bajo ninguna circunstancia. En 2012, a partir de la sanción de la ley 26.842 se produjo una reforma sustancial del delito de trata de personas y en aquellas disposiciones que castigaban el proxenetismo (Arts. 125 bis y siguientes). La reforma directamente eliminó la distinción entre personas mayores y menores de edad, se eliminaron los medios comisivos y se elevó la condena a una pena de 4 a 8 años. La redacción actual del art. 145 bis quedó como sigue le corresponderá dicha pena "al que ofreciere, captare trasladare, recibiere o acogiere personas con fines de explotación, ya sea dentro del territorio nacional, como desde o hacia otros países, aunque mediare el consentimiento de la víctima". La situación de vulnerabilidad como medio comisivo configura un supuesto de agravamiento de la pena del Art. 145 ter, que será de cinco (5) a diez (10) años.
}

${ }^{7}$ La resolución MJSyDH 2198/2008 creó la Oficina de Rescate y Acompañamiento a las personas damnificadas por el delito de trata y ésta fue modificada en 2012 a través de la Resolución MJyDH 731/2012 que puso en funcionamiento el Programa Nacional de Rescate y Acompañamiento de las personas damnificadas por el delito de trata.

\footnotetext{
${ }^{8}$ Desde el año 2008 se sancionaron múltiples legislaciones que fueron clandestinizando distintas etapas para el ejercicio del trabajo sexual. Por citar un ejemplo, el Decreto 936/2011 para la erradicación de la difusión de mensajes e imágenes que estimulen o fomenten la explotación sexual cuyo texto prohibió los avisos que promuevan la oferta sexual o hagan explícita o implícita referencia a la solicitud de personas destinadas al comercio sexual, por cualquier medio, con la finalidad de prevenir el delito de Trata de Personas con fines de explotación sexual y la paulatina eliminación de las formas de discriminación de las mujeres. Asimismo, quedan comprendidos en este régimen todos aquellos avisos cuyo
} Revista del Laboratorio Iberoamericano para el Estudio Sociohistórico de las Sexualidades https://doi.org/10.46661/relies.4994 
contenido pedagógico hacia "las nuevas masculinidades" bajo el lema: "los hombres de verdad no consumen prostitución".

Esta campaña no solo se nutrió del carácter ambiguo de la definición del delito de trata sexual, sino que tal carácter fue la condición de posibilidad para la proliferación de leyes, actos administrativos, organismos especializados y ámbitos de institucionalización del feminismo abolicionista cuya definición de la "experiencia" femenina9 (MacKinnon, 1982; 1995) ha sido muy relevante para la configuración de lo que se ha dado en llamar el "paradigma de la violencia de género" . Este último, refiere a la "violencia de género" ${ }^{10}$ como una categoría político-jurídica que ha promovido formas de intervención, interpretación y tratamiento específicas para abordar las múltiples opresiones a las que están expuestas las mujeres cis; estas formas son utilizadas también para leer las opresiones de otras identidades de género, lesbianas, bisexuales, intersex, trans o travestis, con efectos muy criticables (Trebisacce, 2020, p. 120).

Inserta en este paradigma, la trata de mujeres con fines de explotación sexual ha ocupado un lugar preponderante en la agenda de derechos humanos a nivel global desde finales de la década del noventa del siglo pasado (Miller, 2004: 21). Su consolidación en el ámbito internacional describe un proceso en el cual la participación del feminismo de la gobernanza (Halley et.al., 2006: 340) fue crucial a la hora de problematizar la trata de personas no sólo a nivel internacional, sino por la capacidad de influencia que tuvo en los contextos locales.

Elizabeth Bernstein (2010; 2012), retomando las críticas del feminismo postcolonial señaló el carácter complejo de las alianzas entre feministas (neo)abolicionistas, autoridades religiosas y ONGs movilizadas para empoderar a las mujeres subalternizadas del sur-global en el contexto de la gobernanza feminista de la trata (Halley et.al., 2006; Kapur, 2005; Kempadoo y Doezema, 1998). La autora señala que esta particular forma de gobierno se configura como una de las más relevantes

texto, haciendo referencia a actividades lícitas resulten engañosos, teniendo por fin último la realización de alguna de las actividades aludidas en el párrafo precedente. Este Decreto creó en la órbita del Ministerio de Justicia y Derechos Humanos la Oficina de monitoreo de publicación de avisos de oferta de comercio sexual como autoridad de aplicación.

${ }^{9}$ Dentro del feminismo abolicionista, su gran exponente, Catherine MacKinnon señaló que existe una experiencia común de dominación que hace a todas las mujeres sexualmente subordinadas. Desde este razonamiento del feminismo radical aboga por la disolución de la estructura individualista, naturalista, idealista y moralista del derecho androcéntrico. Para la autora (1995, p. 391) la situación de la mujer en el ámbito jurídico se define mediante la opresión sexista, que es instrumentalizada para describir la verdadera experiencia de todas las mujeres, y es fruto de una premisa que define a la sexualidad femenina como objeto de dominación por parte de los varones. Frente a la alienación y la falsa conciencia que produce este tipo de dominación, MacKinnon (1982, p. 637; 1995, p.187) propone como método la toma de conciencia para poder revelar la verdad que ha sido ocultada por la dominación masculina; esta búsqueda de una verdad trascendente y universal, le permite confiar en la emergencia de una "mujer nueva" que, a través de la toma de conciencia colectiva, pueda trascender las determinaciones del sistema de dominación. En esta propuesta epistemológica, tanto la victimización como la dominación son analizadas como producto de un patriarcado ahistórico, liso y homogéneamente opresivo. En el ámbito internacional, esa influencia explica la notoriedad que adquirió la violencia sexual como una forma genderizada de tortura facilitó la visibilización del fenómeno como propio del ámbito de los derechos humanos, dada su capacidad para describir una situación cuya crueldad nadie podía ignorar. En el ámbito de la ley, esta violencia específica y originaria permitió invocar una opresión común a todas las mujeres del mundo. Junto a la responsabilización de los Estados, este reclamo tenía por finalidad también desplazar las consideraciones respecto de la honestidad de la mujer, a partir de la incorporación de elementos normativos genderizados que pudieran plasmarse en derecho positivo internacional.

${ }^{10}$ En el ámbito de la Organización Naciones Unidas (ONU) a lo largo de la Década de las Mujeres (1975-1985), la violencia fue ocupando un lugar crecientemente relevante dentro de las estrategias para demandar la protección internacional de los derechos humanos. En la Conferencia de Viena de 1993, la violencia contra la mujer se afianzó como una categoría de análisis y de reivindicación -simultáneamente- para abordar las violaciones de derechos humanos de las mujeres, que hasta ese momento eran analizadas como una cuestión de discriminación.

Revista del Laboratorio Iberoamericano para el Estudio Sociohistórico de las Sexualidades https://doi.org/10.46661/relies.4994 
expresiones de lo que ha denominado como "carceral feminism" y que traducimos libremente como feminismo punitivista.

De esta forma, esta contribución se inserta en los desarrollos teóricos que analizan los regímenes anti-trata sexual (Piscitelli, 2015) ${ }^{11}$ como parte de la gubernamentalidad neoliberal, retomando también para el análisis las críticas a la categoría de género en la que se basan las descripciones de las experiencias de explotación sexual ${ }^{12}$ (Abrams, 1995:310) y los análisis relativos a la traducción de las demandas feministas para la protección de los derechos humanos en clave punitiva. Específicamente, en el ámbito del Protocolo, el feminismo (neo)abolicionista definió a la trata sexual como la emergencia de una nueva/moderna esclavitud, como la manifestación más violenta del patriarcado, y señaló la falsa conciencia de quienes consienten a la prostitución, e insistió en los daños irreparables que aquella genera. Asignadas estas características a la explotación sexual, el perfil de la víctima de trata sexual es una cismujer que ha sido desposeída de su dignidad y su libertad, a través del proceso de sometimiento a la prostitución, aun con su consentimiento.

Con relación a ello, queremos retomar lo señalado por Tamar Pitch (2003:256) al momento de analizar los vaivenes en el camino hacia la tipificación de la violencia sexual que ya no sea el resultado del paradigma de la honestidad. La autora le presta particular atención a las contradicciones entre la necesidad de visibilizar la especificidad de la violencia sexual y lograr traducirla a una norma penal. Tal especificidad estuvo determinada por una definición que tenía origen en el placer femenino que sólo existía en tanto opresión y humillación; un ámbito en el cual la libertad sexual es solo disfrutable por los (cis)varones. La criminóloga italiana se pregunta por el sentido y la función que puede cumplir un derecho penal que pudiera distinguir únicamente entre violación y heterosexualidad no violenta. Si nunca podemos hablar de libre consentimiento en las relaciones sexuales por parte de la mujer, ¿cuál podría ser la importancia de un derecho penal que como concepto de delito parta, precisamente de la ausencia de consentimiento?

La criminalización de la demanda constituye un caso paradigmático de la responsabilización de un sujeto que toma decisiones racionales (Bernstein 2012; Hoang y Parreñas 2014; Sanders, et.al, 2009; Saunders y Soderlund, 2003; Weitzer, 2011). Esta individualización, junto al pánico sexual en la que se desarrollan las políticas contra la trata, rediseñan las respuestas en clave punitiva frente a tan variadas situaciones como la pobreza, las migraciones, los derechos laborales, la seguridad y la violencia (Bernstein, 2012; Chapkis 2003; Weitzer, 2011).

De la mano de estas definiciones, llevaremos a cabo un análisis de la criminalización del cliente como una parte sustancial del discurso anti-trata y de las políticas que se diseñaron en ese ámbito, anticipando que estas no consisten en novedosas intervenciones con el objetivo de defender bienes o personas ya existentes, sino que crean nuevas esferas de gubernamentalidad (Valverde, 2010:4). Esta afirmación impide que la definición de explotación sexual pueda ser analizada como algo

\footnotetext{
${ }^{11}$ Aquí retomamos la expresión acuñada por la autora de "regímenes anti-trata" que alude a la constelación de políticas, normas, discursos, conocimientos y leyes sobre trata de personas, formuladas en el entrelazamiento de planos supranacionales, internacionales, nacionales y locales.

${ }^{12}$ Las definiciones del feminismo radical para luchar contra la prostitución, la pornografía y el acoso sexual comenzaban a operar a nivel legal, pero también como la imagen que las mujeres construían de sí. Este feminismo concibe la sexualidad de la mujer como el espacio paradigmático de opresión y como constitutivo de la definición de género de la que parte, tal como expresa Ti-Grace Atkinson, cuando afirma que no hay ninguna feminista digna de tal pertenencia quien, obligada a escoger entre la libertad y el sexo, se incline por este último.
} 
estático en el ámbito de la ley y, por el contrario, su carácter dúctil resulta inestimable para poder enmarcar ${ }^{13}$ (Butler, 2010:24) esas prácticas.

\section{La situación de vulnerabilidad}

Aquí pretendemos analizar el dispositivo (neo)abolicionista, no sólo a través de los resultados de la implementación de sus políticas en clave de aumento de la criminalización de cuerpos feminizados en el mercado sexual (Varela, 2013), sino también desde la necesidad de denunciar las racionalidades que habilitan su expansión a pesar de sus fracasos.

En este sentido, dicho dispositivo orienta simbólicamente la conducta de las nuevas masculinidades y además se proyecta sobre la conducta de las mujeres en situación de vulnerabilidad a través del uso de técnicas de empoderamiento que no son estrictamente regulatorias sino coercitivas (Bernstein, 2010; Saunders y Soderlund, 2003). Por su parte, Laura Agustín (2007) acuñó el término "industria del rescate" para denunciar la capacidad expansiva de la definición de trata sexual, cuyo sostenimiento dependerá de que haya un número creciente de mujeres que se asuman como víctimas, incluso a través de la amenaza del castigo. Esto explica también las razones en virtud de las cuales la trata sexual se ha convertido en una commodity en la competencia por recursos económicos de Estados y ONGs, como así también en reconocimiento político y académico, tal como lo demuestra el sistema de premios y castigos establecidos por agencias estadounidenses en los informes anuales del Trafficking in Persons Report, que condiciona el financiamiento de los programas de lucha contra este delito al cumplimiento del juramento de abstención para promover la industria sexual que deben asumir quienes aspiran a beneficiarse de esos fondos ${ }^{14}$ (Varela, 2015).

Por lo tanto, es conveniente analizar las contradicciones aparentes del gobierno (neo)abolicionista de la trata sexual a partir de la categoría juridicial de la situación de vulnerabilidad, dado que permite dar cuenta de los efectos que generan las políticas de rescate cuando se orientan hacia una "víctima de trata", que actúa sin agencia y cuyo "empoderamiento" se produce a través de

\footnotetext{
${ }^{13}$ La acción de enmarcar remite específicamente a los desarrollos relativos a la precariedad efectuados por Judith Butler. En este sentido, para esta autora, los marcos son esquemas móviles de inteligibilidad, y permiten entonces incorporar al análisis la existencia de las versiones minoritarias de la norma, es decir, enmarcar el marco, permite cuestionarlo mediante la visibilización de las resistencias frente a los violentos intentos normalizadores. En Marcos de guerra, Butler utiliza en numerosas ocasiones el término vulnerabilidad, y aparece casi siempre en relación con el de precariousness, es decir el aspecto corporal de la precarity (en español para distinguir ambos conceptos se recurrió a precariedad para hacer referencia al primero de los conceptos y precariedad para el citado en último lugar). Ahora bien, lo que queremos puntualizar es que, a diferencia de la vulnerabilidad, la precariedad explica el carácter social de la vida, es decir, no se trata de la exposición de nuestro cuerpo a una pérdida o a la violencia, sino al hecho de que nuestra vida depende de otrxs próximos o no. De todos modos, cabe destacar que ninguno de estos conceptos existe por fuera de lo político, de lo social. El "ser" del cuerpo al que se refiere esta ontología es un ser que siempre está entregado a otrxs: a normas, a organizaciones sociales y políticas que se han desarrollado históricamente con el fin de maximizar la precariedad para unxs y de minimizarla para otrxs. Esa relación con otrxs permite la emergencia del sujeto, siempre dependiente (precario) y expuesto a Ixs otrxs (vulnerable). Precariedad y precariedad son conceptos que mantienen una relación de intersección: "Las vidas son por definición precarias: pueden ser eliminadas de manera voluntaria o accidental, y su persistencia no está garantizada de ningún modo. En cierto sentido, es un rasgo de toda vida, y no existe una concepción de la vida que no sea precaria, [...] La precariedad designa esa condición políticamente inducida en la que ciertas poblaciones adolecen de falta de redes de apoyo sociales y económicas y están diferencialmente más expuestas a los daños, la violencia y la muerte [...]". (Butler, 2010: 46).
}

${ }^{14} \mathrm{~A}$ partir de esta cláusula muchas organizaciones han dejado de recibir financiamiento. Las denuncias más contundentes surgieron de parte de organizaciones que venían trabajando en políticas de reducción de daños de HIV/SIDA con trabajadorxs sexuales, dado que esas actividades, repartir preservativos, talleres de acceso a la salud integral, etc. comenzaron a ser interpretadas como promotoras del trabajo sexual.

Revista del Laboratorio Iberoamericano para el Estudio Sociohistórico de las Sexualidades https://doi.org/10.46661/relies.4994 
mecanismos que no logran materializarse por fuera del recurso a la violencia estatal (HannahMoffat, 2004; 2010).

Con base en estas definiciones, el rescate de las víctimas funciona frente a alguien a quien no puede imputársele responsabilidad en el hecho de la explotación sexual, pero a quien sí se le exige que desarrolle herramientas para disminuir la situación de vulnerabilidad en la que se encuentra (Kotiswaran, 2014: 365). Sin embargo, esta modalidad de la trata no se encuentra definida ni en el Protocolo, ni en los ordenamiento jurídico-penales locales ${ }^{15}$. De acuerdo con los travaux préparatoires ${ }^{16}$, la vulnerabilidad hace referencia a cualquier situación en que una persona no tiene otra alternativa que someterse a la explotación. Varios años después, a partir de la interpretación de un instrumento de 1930, la OIM sugirió que la vulnerabilidad debía identificarse con un trabajador económicamente vulnerable que no puede negarse a cumplir con las demandas de su empleador debido al riesgo de perder el empleo.

El grupo de trabajo de Trata de Personas en el ámbito de las Naciones Unidas concluyó que las asociaciones de la vulnerabilidad con un estatus (edad, género, capacidad, situación migratoria irregular) raramente consideran a la vulnerabilidad económica como un factor determinante. En tales documentos, se demuestra cierta preocupación por la falta de atención suficiente a esta última como un indicador que permitiría dotar de significado a la vulnerabilidad, y reubicarla de ese modo en el ámbito de explotación ${ }^{17}$. Sin embargo, desplazar la situación de vulnerabilidad hacia la explotación económica no funciona en la trata sexual, debido al postulado abolicionista que identifica la prostitución con la explotación. No obstante, con base en esta ecuación extensiva, no todas cuentan como víctimas. Una de las principales tareas de las políticas anti-trata es la de establecer un parámetro universalizable para identificar a las verdaderas víctimas y distinguirlas de otras mujeres responsables de su propia explotación.

Frente al esquema planteado por la gobernanza (neo)abolicionista de la trata sexual las tecnologías de gobierno de la trata tienen como objetivo empoderar a las mujeres frente a la explotación sexual. Sin embargo, la situación de vulnerabilidad opera como una condición de posibilidad para que el empoderamiento se convierta en coercitivo/paternalista; y también como fuente de legitimidad al (neo)abolicionismo, que se presenta como síntesis superadora de otros modelos de gobierno de la prostitución.

El (neo)abolicionismo compele a la protección de las mujeres frente a la amenaza de la explotación sexual de un amplio espectro de "tercerxs", incluido el cliente, además incrementa y diversifica la vigilancia sobre las formas de vida de las trabajadoras sexuales (cis, trans y travestis) mujeres. En este contexto, pueden observarse las complejas imbricaciones entre el tendencial abandono del modelo disciplinario y las actuales formas de control que determinan el abandono del "daño" como respaldo de las imputaciones delictivas y la emergencia de la "vulnerabilidad" como forma de legitimar la intervención con carácter moralizante (Harcourt, 1999:112).

\footnotetext{
${ }^{15}$ Anecdóticamente, la situación de vulnerabilidad fue incorporada al Protocolo de Palermo contra la trata a último momento, como un intento de consensuar una definición de trata que pudiera transcender los debates sobre la prostitución.

${ }^{16}$ Report of the Ad Hoc Committee on the Elaboration of a Convention Against Transnational Organized Crime on the Work of Its First to Eleventh Session-Addendum: Interpretative Notes for the Official Records (Travaux Préparatoires) of the Negotiation of the United Nations Convention Against Transnational Organized Crime and the Protocols Thereto, U.N. Doc. A/55/383/Add.1. (3/11/ 2000)

${ }^{17}$ Grupo de Expertos en Trata de Personas (2012) Issue Paper on Abuse of a Position of Vulnerability and Other "Means" Within the Definition of Trafficking.
}

Revista del Laboratorio Iberoamericano para el Estudio Sociohistórico de las Sexualidades https://doi.org/10.46661/relies.4994 
Ahora bien, ¿qué sucede cuando nos encontramos con mujeres que se resisten a ser empoderadas bajo estas tecnologías? Las resistencias de las mujeres subalternizadas demuestran el carácter inestable de las formas de gobernanza y las relaciones de poder que proliferan en ese ámbito bajo la organización dicotómica propia de los discursos neoliberales y legales. Frente a ello, el dispositivo funciona gracias al silenciamiento de las voces de Ixs trabajadorxs sexuales producido por la situación de vulnerabilidad, ya que éste determinará cuándo una decisión es válida; es decir, la vulnerabilidad (neo)abolicionista custodia el espacio no estriado de las intervenciones que hablan en nombre de las mujeres para asegurar la eficacia del rescate.

El abandono del trabajo sexual (exiting) como respuesta a la situación de vulnerabilidad es la reina de las intervenciones, dado que bajo este esquema logra que cualquier fracaso pueda ser atribuible a la incapacidad de empoderarse de las mujeres (Scoular y O'Neill, 2007).

\section{El silenciamiento de Ixs trabajadorxs sexuales}

El silenciamiento opera bajo una determinada concepción de género de la que parte el (neo)abolicionismo y habilita un conjunto de tecnologías de control funcionales al gobierno (post)disciplinario de la prostitución. En este ámbito, la definición (neo)abolicionista de la situación de vulnerabilidad permite que "la incapacidad de empoderarse de las víctimas de trata sexual" se transforme en el recurso para perpetuar el gobierno de la trata. En este apartado, desarrollamos los métodos de silenciamiento del (neo)abolicionismo a través de lo que Kristie Dotson (2012) define como opresión epistémica y el feminismo negro describe como una ocupación (Hill Collins, 2000; Carby, 1982; Sandoval, 2000).

La categoría de vulnerabilidad del (neo)abolicionismo reside en las asunciones morales y corporales estáticas y esencialistas en las que se sustentan sus privilegios y su definición de opresión. La situación de vulnerabilidad se basa en una definición de género que opera como una categoría totalizadora incapaz de comprender los procesos de racialización, precarización, ni el acoplamiento de distintos sistemas de opresión que describen la situación de sujetos particulares. Las concepciones del feminismo radical establecen una relación entre el cuerpo y la subjetividad que opera epistemológicamente como una negación de las experiencias de Ixs trabajadorxs del sexo y capilarizan los procesos de estigmatización y criminalización de sus vidas y sus decisiones.

Al respecto, Nancy Fraser (1989) sostiene que los esfuerzos entre las mujeres para redefinir sus problemas en sus propios términos compiten o se suprimen por los discursos profesionales. Estos despliegan su poder a través de etiquetar y caracterizar los signos del trauma, educando a las mujeres sobre la verdadera naturaleza de su victimización y autopoíeticamente definir también el éxito de la recuperación (Maqueda, 2017). En estos tratamientos están implícitas las interpretaciones de Ixs expertxs respecto de lo que constituye la vulnerabilidad.

De esta forma, las intervenciones para proteger a las mujeres en el contexto de la trata sexual parten de una definición de empoderamiento que sólo valida como consentimiento el de una mujer capaz de tomar decisiones acertadas, de autogobernarse (Hackett, 2013; Hannah-Moffat, 2000; 2006) Acertadas, en este caso, son aquellas decisiones que apuntan al reconocimiento de la situación de vulnerabilidad en el mercado sexual como algo intrínseco a él, y por tanto el abandono es la única posibilidad de disminuir la exposición a la vulnerabilidad.

El papel de la situación de vulnerabilidad en el contexto de las políticas anti-trata habilita que el continuo entre el gobierno disciplinario de la prostitución y las nuevas tecnologías de rescate se refuercen mutuamente (Brown, 2014, 2015).

En el caso de las víctimas de trata con fines de explotación sexual, el perfil utilizado describe dos situaciones posibles: la víctima encarna un riesgo para sí, al no poder (auto)protegerse de 
situaciones de explotación en virtud de su vulnerabilidad, y simultáneamente, es un riesgo para el éxito de las políticas contra la trata. En el caso de la víctima de este delito estamos frente a un sujeto atravesado por dos categorías en momentos sucesivos y que no pueden ser distinguidas entre sí: 1) si se asumen como trabajadoras sexuales deben ser reprimidas, si bien el código penal no tipifica el ejercicio de la prostitución; 2) como víctimas (el 99\% de ellas según las cifras estratégicas) ${ }^{18}$ deben ser objeto de rescate y ayuda que les permita convertirse en sujetos autónomos, es decir, abandonar el trabajo sexual.

En este sentido, tal como sostiene el Protocolo de actuación de las fuerzas de seguridad ${ }^{19}$, el empoderamiento de la víctima consiste en que desarrolle otras estrategias de supervivencia que no sean el trabajo sexual, que testifiquen en los procesos judiciales y que, en el caso de que se trate de migrantes, retornen a su lugar de origen.

Frente a este epistemicidio, el trabajo de Cecilia Varela (2013) minuciosamente desarma estos presupuestos a partir del análisis de sentencias judiciales y del trabajo de campo con mujeres condenadas por el delito de trata. Su trabajo pone en evidencia que la lucha contra la trata promueve la criminalización de las diferentes formas en que las cis y trans mujeres y travestis, en algunos casos migrantes, organizan el trabajo sexual. Del total de las condenas por este delito, el $43 \%$ corresponden a cismujeres, arrojando una ratio inédita de criminalización, debido a que más que casos de trata sexual estamos frente a definiciones (neo)abolicionistas del mercado sexual.

En las condenas judiciales sobre este delito, el saber experto de quienes intervienen en un proceso de trata sexual describen, con mayor o menor detalle, la vulnerabilidad de las mujeres cis en "el sistema prostibulario". A diferencia de la trata con fines de explotación laboral, no se han diseñado mecanismos que permitan determinar cuándo estamos frente a un hecho punible de explotación, dado que la concepción de género de la que parten les exime de tal esfuerzo ${ }^{20}$.

La vulnerabilidad engloba un conjunto de características -susceptible de ser siempre ampliadoasociadas al ejercicio de la prostitución como un ámbito de victimización per se. Estas interpretaciones jurisprudenciales -ancladas en la premisa abolicionista de que la prostitución siempre es explotación sexual-, habilita la persecución y castigo de las múltiples formas de resistencia a la criminalización de las formas de vida de Ixs trabajadorxs sexuales (v.gr. se criminalizan el intercambio de información a través de redes sociales e incluso, formas muy rudimentarias de organización de la actividad; su afiliación al Sindicato de Trabajadorxs Sexuales [AMMAR] opera como presupuesto para la configuración de la explotación sexual, y se legitima el aumento de la violencia institucional en el espacio público, en especial de mujeres trans o travestis migrantes, entre otros efectos). En este contexto, Ixs trabajadorxs sexuales se ven obligadxs a decidir entre su libertad o su seguridad y en esta clave, articulan estrategias frente a la exposición diferencial a la violencia institucional.

\footnotetext{
${ }^{18}$ Según el Fiscal Titular de la Protex Dr. Marcelo Colombo, el 99\% de los casos del sistema prostibulario son de trata sexual", disponible en http://www.diariofemenino.com.ar/marcelo-colombo-el-99-de-los-casos-del-sistemaprostibulario- son-de-trata-sexual

${ }^{19}$ Resolución 742/2011 del Ministerio de Seguridad, Protocolo de Actuación para las fuerzas federales disponible en http://www.infoleg.gov.ar/infole- gInternet/anexos/185000-189999/185854/norma.htm

${ }^{20}$ De conformidad con la Resolución PGN № 46/2011 "Guía de procedimientos y criterios para detectar e investigar la trata con fines de explotación laboral" cuyo objetivo fue dotar a Ixs operadorxs judiciales indicadores que permitieran distinguir entre tres figuras tipificadas en el Código Penal: la esclavitud (trata), el trabajo forzado y la reducción a la servidumbre o condición análoga.
}

Revista del Laboratorio Iberoamericano para el Estudio Sociohistórico de las Sexualidades https://doi.org/10.46661/relies.4994 
Para abundar en este análisis, resulta interesante comentar brevemente la actuación del Programa Nacional de Rescate y Acompañamiento de las personas damnificadas por el delito de trata del Ministerio de Justicia y Derechos Humanos, y su campaña "sin clientes no hay trata", que publica hasta diciembre de 2015 un total de 10.000 mujeres "rescatadas" y casi 6000 llamadas recibidas a la línea de denuncia anónima \#145 (desde abril de 2008). El Programa tiene por objeto acompañar con asistencia psicológica la actuación de las fuerzas federales de seguridad durante los allanamientos y "preparar" a las víctimas para su "declaración" en sede judicial. Zaida Gatti, la titular del programa, afirmó que "...del total, entre un $60 \%$ y $70 \%$ son víctimas de explotación sexual, es decir que son más de 7000 las mujeres que hemos asistido por este delito de las cuáles solo el 2\% se reconoce como víctima. En proporción, nueve de cada 10 procedimientos corresponde a explotación sexual" (el resaltado nos pertenece) ${ }^{21}$.

Como se desprende de lo anterior, la vulnerabilidad en el delito de trata sexual funciona a partir de asunciones morales y corporales estáticas y esencialistas en las que se sustentan los privilegios y la definición de explotación sexual del feminismo (neo)abolicionista; es decir, las "soluciones" se basan en una definición de género que opera como una categoría totalizadora incapaz de comprender los procesos de racialización, precarización, ni el acoplamiento de distintos sistemas de opresión que describen la situación de sujetos particulares. Esta perspectiva define a la vulnerabilidad como una relación entre el cuerpo y la subjetividad que opera como una negación de las experiencias de Ixs trabajadorxs del sexo abriendo instancias punitivas no contempladas en la ley.

La funcionaria, para explicar el fracaso del rescate, recurre a una estrategia típica del gobierno (neo) abolicionista de la trata: cuando fallan los instrumentos no significa que éstos no sean eficaces, sólo falta un poco más de tiempo, hay que mirar mejor. De esta forma, entiende que la negativa a considerarse "una víctima de trata sexual es un mecanismo de defensa en el cual necesitan de alguna manera no identificarse con esa persona que sufrió violaciones diarias, porque cada cliente es un violador (...) y si la víctima se va, no tiene dónde ir y luego vuelve al prostíbulo. Entonces ella cree falsamente que es libre por no reconocerse como víctima. Ese es el punto crucial. Ella está totalmente disociada y guarda todo eso reprimido en algún sector de su aparato psíquico"22.

Podemos observar que se produce un impasse de la lógica rescatista: no sabemos nada en lo relativo al cumplimiento del capítulo de la ley referido a la garantía de los derechos humanos como forma de compensar la vulnerabilidad. Si todas son víctimas, ¿ por qué no sujetas de derecho?

Si se contrasta "el éxito" de las políticas de rescate del Ministerio de Justicia con los datos de los informes de la PROTEX (2015) ${ }^{23}$ sobre las causas de trata sexual se advierte que con base en las propias percepciones de lxs operadorxs judiciales y de la aplicación del principio de oportunidad, se establece una selectividad "secundaria" de aquellas causas con víctimas adecuadas que pueden prosperar bajo la racionalidad del rescate.

\footnotetext{
${ }^{21}$ Disponible en https://www.tiempoar.com.ar/articulo/view/64584/zaida-gatti-sa-lo-el-2-por-ciento-de-lasexplotadas-sexualmente- se-reconoce-como-va-ctima, (visita:11/2/2017)

${ }^{22}$ https://www.tiempoar.com.ar/articulo/view/64584/zaida-gatti-sa-lo-el-2-por-ciento-de-las-explotadassexualmente- se-reconoce-como-va-ctima, (visita:11/2/2017).

${ }^{23}$ En el año 2013 en el ámbito de la Procuración General de la Nación se creó (Resolución PGN no 805/2013) la "Procuraduría de Trata y Explotación de Personas" que reemplazó a la anterior UFASE (Unidad de Asistencia en Secuestros Extorsivos y Trata de Personas). Entre sus facultades y objetivos se encuentran la de prestar asistencia a las Fiscalías de todo el país en el trámite de las causas por hechos de secuestro extorsivo, trata de personas y delitos conexos a la trata y asistir a la Procuradora General de la Nación en el diseño de la política criminal del Ministerio Público con relación a estos delitos.
}

Revista del Laboratorio Iberoamericano para el Estudio https://doi.org/10.46661/relies.4994

Sociohistórico de las Sexualidades 
En este sentido, en el contexto de la presentación del Programa de Actualización de Género y Derecho en la Facultad de Derecho (UBA), aproveché la presencia del Titular de la PROTEX para plantearle mi inquietud respecto de la falta aplicación de la cláusula de no punibilidad contemplada en el artículo 5 de la ley $26.842^{24}$ para las víctimas del delito de trata, como forma de contrarrestar las altas ratios de criminalización de mujeres por este delito. La pregunta hacía referencia a un artículo doctrinario de su autoría en el cual había reconocido que las "ahora autoras" podrían ser las "antes" de víctimas de trata "reconvertidas" luego de su etapa de sometimiento (Colombo y Mángano, 2011). Me respondió que no había dado la instrucción de aplicar la cláusula de no punibilidad al resto de las fiscalías, tal como establece la normativa que regula la función pública que desempeña, "porque nadie le hubiera hecho caso" (sic).

Aun cuando los informes y las sentencias hagan referencia a factores económicos, sociales o de género, se puede observar cómo los mecanismos de individualización y patologización propios del (neo)abolicionismo, problematizan la explotación sexual como una experiencia traumática que encuentra sus causas en un pasado de abusos y de violencia (Maqueda, 2014:16). Este continuo es instrumentalizado como la causa y el origen de la situación de prostitución y sirve como antídoto frente al consentimiento. Dentro de las tecnologías del rescate, la victimización no solo es un indicador de eventos pasados, sino fundamentalmente de riesgo futuro.

¿Se puede afirmar entonces que la necesidad de un tratamiento prolongado para que la mujer se reconozca como víctima, que según Zaida Gatti requiere más tiempo que aquel que demanda la elevación a juicio oral, es la razón por la cual se condena a las mujeres que todavía no alcanzaron ese objetivo?

¿Las mujeres, sujetos de protección, son condenadas porque no son buenas víctimas? La cláusula de no punibilidad podría mitigar esta situación, pero el saber experto siempre aspira a mirar mejor.

En este sentido, la vulnerabilidad de la mujer como concepto normativo central en la problematización de la trata, transforma los cuerpos en un espacio abierto a la explotación. Este elemento se convierte en el eje de la definición del delito de trata, cuyo particular presupuesto de partida, no solo despoja de la capacidad de autonomía, sino que termina por transformar a los sujetos en peligrosos (Castel, 1999). La desafortunada ecuación entre la sexualidad y la agencia con base en la que se decide la intervención de la industria del rescate configura prácticas sexistas, clasistas y neocoloniales. El obstáculo epistemológico produce que quienes están encargadxs de diseñar las intervenciones no puedan contemplar que muchos de los elementos descritos como la exposición a la vulnerabilidad generada por "la prostitución", son en realidad regímenes específicos de criminalización y denigración que marginan y oprimen a Ixs trabajadorxs sexuales (Zatz, 1997:289).

Frente al autoritarismo del silenciamiento hay que seguir denunciando los métodos de intervención de la ley penal sobre la vida de Ixs trabajadorxs sexuales 0 , en otras palabras, la relación contingente y compleja entre los aspectos formales y materiales de la ley y las múltiples formas en las que el sistema legal es diseñado para moldear esa relación, pero nunca controlarla completamente. Este es un aspecto fundamental de la crítica al control penal y que es ignorada por el feminismo (neo)abolicionista y aprovechada por quienes obtienen beneficios materiales e inmateriales de la lucha contra la trata.

\footnotetext{
${ }^{24} \mathrm{El}$ artículo establece que: "Las víctimas de la trata de personas no son punibles por la comisión de cualquier delito que sea el resultado directo de haber sido objeto de trata.Tampoco les serán aplicables las sanciones o impedimentos establecidos en la legislación migratoria cuando las infracciones sean consecuencia de la actividad desplegada durante la comisión del ilícito que las damnificara".
}

Revista del Laboratorio Iberoamericano para el Estudio https://doi.org/10.46661/relies.4994

Sociohistórico de las Sexualidades 
Los derechos humanos, en conclusión, se convierten en el patrimonio exclusivo de aquellas mujeres que performan adecuadamente la normatividad inscrita en el género que impulsa el (neo)abolicionismo. Para el resto de las mujeres, solo queda una línea discontinua de mecanismos de intervención represivos que ahondan en la vulnerabilidad. 


\section{Bibliografía}

Abrams, K. (1995). "Sex Wars Redux: Agency and Coercion in Feminist Legal Theory". Columbia Law Review. Vol. 304:304-376.

Agustin, L. (2007). Sex at the Margins: Migrations, Labour markets and the Rescue Industrie. Zed Books: London.

Ammar/Daich, D y Varela, C.I. (2014). Políticas antitrata y vulneración de derechos de las Trabajadoras Sexuales, en http://www.ammar.org.ar/IMG/pdf/informe-ammar.pdf Accedido el 12 de diciembre de 2014).

Ammar /RedTraSex. DAICH, D. y VARELA, C.I. (2016). Trabajo Sexual Y Violencia Institucional: Vulneración De Derechos Y Abuso De Poder Hacia Mujeres Trabajadoras Sexuales. En http://www. redtrasex.org/IMG/pdf/informe_nacional_argentina.pdf Accedido el 30 de diciembre de 2016.

Amnesty International. (2016). "Lo que hago no es un delito". El coste humano de penalizar el trabajo sexual en la Ciudad de Buenos Aires, Argentina. https://www.amnesty.org/es/documents/ amr13/4042/2016/es.

Andrijasevic, R. (2010) Migration, Agency and Citizenship in Sex Trafficking. Palgrave Macmillan: New York.

Aradau, C. (2004). The Perverse Politics of Four-Letter Words: Risk and Pitty in the Securiti- zation of Human Trafficking. Millenium Journal of International Studies 22(2): 251-277.

Aradau, C. (2008). Rethinking Trafficking in Women. Politics of Security. Palgrave: London.

Bernstein, E. (2010). Militarized humanitarianism meets carceral feminism: the politics of sex, rights, and freedom in contemporary antitrafficking campaigns. Signs: Journal of Women in Culture and Society 36(1): 45-71.

Bernstein, E. (2012). Carceral politics as gender justice? Theory and Society 41: 233-59.

Brown, K. (2014). Questioning the vulnerability zeitgeist: care and control practices with 'vulnerable young people'. Social Policy and Society13(3): 371-87.

Brown, K. (2015). Vulnerability and Young People: Care and Social Control in Policy and Practice. Policy Press: Bristol.

Bumiller, K. (2008). In an abusive state. Duke University Press: Durkham.

Butler, J. (2010). Marcos de guerra. Las vidas Iloradas. Paidós: México.

Carby, H. (1982). White Woman Listen! Black Feminism and the Boundaries of Sisterhood En The Empire Strikes Back: Race and Racism in 70s Britain. The Centre for Contemporary Cultural Studies: London.

Castel, R. (1991). From dangerousness to risk, en Burchel, Graham, et. al (eds.). The Foucault effect. Studies on Governmentality. Chicago University Press. Chicago:281-298.

Chapkis, W. (2003). Trafficking, Migration, and the Law: Protecting Innocents, Punishing Inmigrants. Gender and Society 17(6): 923-937.

Colombo, M. y Mangano, A. (2011). El consentimiento de la víctima en la trata de personas y un análisis sobre los medios comisivos previstos en la figura penal. En http:// www.mpf.gov.ar/Accesos/Ufase/consentimiento_victima_trata.pdf Accedido el 12 de marzo de 2014. 
Dedillet, S. y Östergren, P. (2012). La ley sueca sobre la compra de sexo: presuntos éxitos y resultados demostrables, en Villacampa Estiarte, Carolina(coord.) Prostitución chacia la legalización? Tirant lo Blanch. Valencia: 113-145.

Ekberg, G. (2004). The Swedish Law that Prohibits the Purchase of Sexual Services. Best Practices for Prevention of Prostitution and Traffkicing in Human Beings. Violence against Women 10: 1187-1218.

Foucault, M. (1984). El juego de Michel Foucault, en Saber y verdad. Ediciones de la Piqueta: Madrid: 127-162.

Fraser, N. (1989). Unruly practises: power discourse and gender in contemporary social theory. University of Minnesota Press: Minnesota.

Gatti, Z. (2013). Las víctimas de la Trata. Política de restitución de derechos. El Programa Nacional de Rescate y Acompañamiento a las Personas Damnificadas por el Delito de Trata. Trata de Personas. Políticas de Estado para su prevención y sanción. Ministerio de Justicia y Derechos Humanos. Infojus: Buenos Aires.

Grupo de Expertos en Trata de Personas (2012) Issue Paper on Abuse of a Position of Vulnerability and Other "Means" Within the Definition of Trafficking.

Hackett, C. (2013). Transformative Visions: Governing Through Alternative Practices and Therapeutic Interventions at a Women's Reentry Center. Feminist Criminology 8(3): 221-242.

Halley, J.; Kotiswaran, H.S.; Thomas, Ch. (2006). From The International To The Local In Feminist Legal Responses To Rape, Prostitution/Sex Work, And Sex Trafficking: Four Studies In Contemporary Governance Feminism. Harvard Journal of Law and Gender 29: 336-423.

Hannah-Moffat, K. (2000). Prisons that Empower: Neoliberal Governance in Canadian Women's Prisons. British Journal of Criminology 40: 510-531.

Hannah-Moffat, K. (2006). Pandora's Box: Risk/Need and Gender-Responsive Corrections. Criminology and Public Policy 5(1): 183-192.

Harcourt, B. (1999). The collapse of Harm Principle. Journal of Criminal Law and Criminology 90(1): 109-194.

Hill Collins, P. (2000). Black Feminist Thought: Knowledge, Consciousness, and the Politics of Empowerment, 2nd ed.Routledge: New York.

Hoang, K.K y Salazer Parreñas, R. (2014). Human Trafficking Reconsidered. Rethinking the Problem, Envisioning new Solutions. The International Debate Education Association: New York.

Iglesias Skuli, A. (2013). La trata de mujeres con fines de explotación sexual. Didot: Buenos Aires.

Kelly, L. (2005). You can find anything you want: A critical reflection on research on trafficking in persons within and into Europe. International Migration 43(1-2): 235-65.

Kempadoo, K. y Doezema, J. (1998). Global Sex Workers: Rights, Resistance, and Redefinition. Routledge: New York.

Kapur, R. (2005). Erotic Justice: Law and the New Politics of Postcolonialism.The Glass House Press: London.

Kotiswarn, P. (2014). Beyond Sexual Humanitarianism: A Postcolonial Approach to Anti-Tra- fficking Law. Ucirvine Law Review 4: 353-406.

Kulick, D. (2003). Sex in the New Europe: The Criminalization of Clients and Swedish Fear of Penetration. Anthropological Theory 3(2): 199-218. 
Makinnon, C. (1983). Feminism, Marxism, Method And State: Towards A Feminist Jurisprudence, Signs, 8 (2): 635-58.

Makinnon, C. (1995). Hacia una teoría feminista del Estado. Cátedra: Valencia.

Maqueda Abreu, M.L. (2014). El peso del género y otras identidades culturales en la criminalización de las mujeres. TransJus Working Paper 4: 1-34.

Maqueda Abreu, M.L. (2017). La prostitución: el "pecado" de las mujeres. Cuadernos Electrónicos de Filosofía del Derecho 35: 64-89.

Miller, A. (2004). Sexuality, Violence Against Women, and Human Rights: Women Make Demands and Ladies Get Protection. Health and Human Rights 7(2): 16-47.

Munro, V.E. y Scoular, J. (2012). Abusing Vulnerability? Contemporary Law and Policy Responses to Sex Work in the UK. Feminist Legal Studies 20 (3): 189-206.

OIT.(2005).Una alianza global contra el trabajo forzoso. Informe Ib, Conferencia Internacional del Tra- bajo 93va., Ginebra.

OIT.(2007). Informe III (Parte Ib): Erradicar el trabajo forzoso. Estudio General Relativo al Convenio sobre el trabajo forzoso, 1930 (No.29), y al Convenio sobre la Abolición del Trabajo Forzoso, 1957 (No.105). Conferencia Internacional del Trabajo, 96ta. Ginebra.

Ounida. (2003). Trabajo sexual y VIH/SIDAstonUSIDA. 2012. The HIV and Sex Work Collection Innovative responses in Asia and the Pacific.

Pisticelli, A. (2015). Riesgos: la capilarización del enfrentamiento a la trata de personas en las tensiones entre planos supranacionales, nacionales y locales. Texto preparado para el IV Congreso Latinoamericano sobre Trata y Tráfico de Personas. En https://www.academia.edu/24995943/Riesgos_la_capilarización_del_enfrentamiento_a_la_trata _de_personas_en_las_tensiones_entre_planos_supranacionales_nacionales_y_locales. Accedido el 22 de julio de 2019.

Pitch, T. (2003). Responsabilidad Limitadas. Actores, Conflictos y Justicia. Adhoc: Buenos Aires.

Report of the Ad Hoc Committee on the Elaboration of a Convention Against Transnational Organized Crime on the Work of Its First to Eleventh Session-Addendum: Interpretative Notes for the Official Records (Travaux Préparatoires) of the Negotiation of the United Nations Convention Against Transnational Organized Crime and the Protocols Thereto, U.N. Doc. A/55/383/Add.1. (3/11/2000)

Sandoval, Ch. (2000). Methodology of the Oppressed. University of Minnesota Press: Minneapolis. Sanders, T.; O' Neill, M. y Pitcher, J. (2009). Sex Work, Policy and Politics. Sage Publishing: London.

Saunders, P. y Soderlund, G. (2003). Threat or Opportunity? Sexuality, Gender The EEB, and Flow of Trafficking Discourse. Canadian Women Studies 22(22): 16-24.

Scoular, J. (2004). The Subject of Prostitution: Interpreting the Discursive, Symbolic, and Material Position of Sex Work in Feminist Theory. Feminist Theory 5(3): 343-355.

Scoular, J. y O'Neill, M. (2007). Regulating Prostitution. Social Inclusion, Responsibilization and the Politics of Prostitution Reform. British Journal of Criminology 47(3): 764- 778.

Trebisacce Marchand, C. (2020). Un nacimiento situado para la violencia de género. Indagaciones sobre la militancia feminista porteña de los años 80. Anacronismo e Irrupción 10(18): 118-138.

Valverde, M. (2010). Questions of Security: A Framework for Research. Theoretical Criminology 15: 3-22.

Revista del Laboratorio Iberoamericano para el Estudio Sociohistórico de las Sexualidades https://doi.org/10.46661/relies.4994 
Varela, C.I. (2013). De la "letra de la ley" a la labor interpretante: la "vulnerabilidad" femenina en los procesos de judicializacion de la ley de trata de personas (2008-2011). Cadernos Pagu 41:265302. En http://www.scielo.br/pdf/cpa/n41/15.pdf Recuperado el 12de noviembre de 2016.

Varela, C.I. (2015). La campaña antitrata en la Argentina y la agenda supranacional, en Daich, D. y Sirimarco, M. (Coords), Género y violencia en el mercado del sexo. Política, policía y Prostitución. Biblos: Buenos Aires: 109-149

Weitzer, R. (2011). Legalizing Prostitution: From Illicit Vice to Lawful Business. New York University Press: New York.

Zatz, N. (1997). Sex Work/Sex Act: Law, Labor, and Desire in Construction of Prostitution. Signs Journal of Women Culture and Society 21(2): 227-308. 